much lower. In several countries, like Angola, little is known about this infectious neglected disease. In the 1970s, a group of Portuguese researchers described three cases of cutaneous leishmaniasis in children from Huambo district and in the 1990s visceral leishmaniasis was diagnosed in an African patient. More recently a canine survey in Luanda revealed two Leishmania-infected dogs.

After some suspected cases of human cutaneous leishmaniasis in Huambo region in 2017, the Angola health authorities and the Instituto de Higiene e Medicina Tropical (IHMT), Lisbon, Portugal, established a collaboration to analyse samples from some suspected cases.

Methods Three paraffin-embedded human skin samples from dermatological lesions were sent to IHMT for molecular analysis. After DNA extraction, PCR was performed by using four protocols with different molecular markers.

Results One PCR protocol using a nested approach was positive in two of the samples. Sequencing analysis confirmed Leishmania sp. DNA.

Conclusion This was the first time that suspected human cutaneous samples were screened for leishmaniasis by molecular methods with detection of Leishmania sp. DNA. These preliminary studies highlight the need for higher awareness of health professionals for leishmaniasis clinical forms, to recognise risk factors and the epidemiological features of leishmaniasis in the Huambo province. It would be relevant to perform further epidemiological studies to confirm if this vector-borne disease could be emergent in this country.

\section{PO 8515 CAPACITY BUILDING IN PREPARATION FOR AN HIV VACCINE TRIAL: THE GLOBALLY RELEVANT AIDS VACCINE EUROPE-AFRICA TRIALS PARTNERSHIP (GREAT)}

\begin{abstract}
${ }^{1}$ William Kilembe*, ${ }^{2}$ Brenda Okech, ${ }^{3}$ Leslie Nielsen, ${ }^{3}$ Vincent Muturi-Kioi, ${ }^{4}$ Walter Jaoko, ${ }^{4}$ Gaudensia Mutua, ${ }^{5}$ Eduard Sanders, ${ }^{2} J u l i e t ~ M p e n d o, ~{ }^{3}$ Anne Gumbe, ${ }^{3}$ Kundai Chinyenzi, ${ }^{3}$ Jan De Bont, ${ }^{3}$ Hester Kuipers, ${ }^{8}$ Alison Crook, ${ }^{6}$ Deborah King, ${ }^{7}$ Pontiano Kaleebu, ${ }^{3}$ Patricia Fast, ${ }^{8}$ Tomáš Hanke. 'Zambia Emory HIV Research Project, Lusaka, Zambia; ${ }^{2}$ Uganda Virus Research Institute - International AIDS Vaccine Initiative; ${ }^{3}$ International AIDS Vaccine Inititative, New York, USA; ${ }^{4}$ University of Nairobi, Kenya; ${ }^{5}$ Kenya Medical Research Institute, Kenya; ${ }^{6}$ International AIDS Vaccine Initiative - Human Immunology Laboratory, Nairobi, Kenya; ${ }^{7}$ Medical Research Council - Uganda Virus Research Institute, Uganda; ${ }^{8}$ University of Oxford, UK
\end{abstract}

10.1136/bmjgh-2019-EDC.126

Background The Globally Relevant AIDS Vaccine EuropeAfrica Trials (GREAT) partnership is an EDCTP-funded project that aims to foster collaboration between institutions in Europe and sub-Saharan Africa to build capacity among African clinical research centres (CRCs) for the design and conduct of HIV-1 vaccine efficacy trials.

Methods In January 2017, the University of Oxford (UOXF) and five CRCs in Kenya, Uganda and Zambia were awarded a 5 year grant for capacity building and to support conduct of an HIV-1 vaccine trial in different high-risk populations across Africa using cross-clade (conserved protein regions) T-cell vaccines. UOXF and CRCs embarked on activities to strengthen capacity of the CRCs for future efficacy trials. This included training, community engagement, cohort preparation and infrastructure upgrade.

Results In the first year, the African investigators at the CRCs collaborated on the development of a protocol aimed at assessing the safety and immunogenicity of the tHIVconsvX vaccines. In preparation for the planned vaccine trial, infrastructure upgrades were prioritised at all partner sites and this included building laboratory space and procurement of appropriate laboratory equipment. Planned infrastructure upgrades will also ensure that high-risk populations can be safely and confidentially included in HIV prevention clinical trials. Systematic community engagement was implemented at all sites, training in GCP/GCLP was provided and training is planned for nominated CRC staff to lead community engagement efforts.

Conclusion Improved infrastructure and the provision of targeted training will enhance future trials and increase the capacities of CRCs and staff to conduct quality trials in previously hard-to-reach populations. Early collaboration between investigators from European and sub-Saharan African institutions, with equal responsibilities in the protocol development process, established a meaningful partnership. EDCTP funding also offers a unique opportunity for capacity building.

\section{PO 8518 LATENT TUBERCULOSIS INFECTION DETECTION BY IGRA USING MYCOBACTERIUM TUBERCULOSIS ALTERNATIVE DESATURASE A1 IMMUNE-DOMINANT ANTIGEN}

${ }^{1}$ Niaina Rakotosamimanana*, ${ }^{2}$ Mark Doherty, ${ }^{1}$ Vaomalala Raharimanga, ${ }^{1}$ Vincent Richard, 'Brigitte Gicquel, ${ }^{3}$ Alimuddin Zumla, ${ }^{1}$ Voahangy Rasolofo. ${ }^{1}$ Institut Pasteur de Madagascar, Antananarivo, Madagascar; '2GlaxoSmithKline, London, UK; ${ }^{3}$ University College London, UK

\subsection{6/bmjgh-2019-EDC.127}

Background Despite an efficient treatment and a widely-used vaccine, one third of the world's population is estimated to be latently infected (LTBI) with Mycobacterium tuberculosis (Mtb) and are at risk of progressing to contagious active tuberculosis. New TB vaccines and improved detection of LTBI are therefore urgently required for global TB control. As a first step towards these goals, a better understanding of host recognition and response to immunogenic Mtb antigens is needed.

Methods In this study, interferon- $\gamma$ release in response to the immunodominant antigens ESAT-6, TB10.4, Ag85A, Rv2031 and DesA1 was assessed by ELISPOT on PBMC from 55 newly diagnosed pulmonary TB patients, 121 of their household contacts and 123 matched community controls, enrolled in a high-burden area.

Results ESAT-6 and PPD ELISPOT responses were higher in the TB patient group compared to both contacts and controls $(p<0.05$ repectively). These responses increased with time after recruitment in the contacts and fell after successful treatment in the patients consistent with the hypothesis that responses to these antigens reflect antigenic or bacterial load. However, the response to DesA1 was significantly lower in the controls compared to the contacts $(\mathrm{p}<0.05$ respectively). Receiver Operating Characteristic curve analysis showed that PPD/ ESAT-6 best segregated TB patients from the other groups, while DesA1 best segregated contacts from controls.

Conclusion The present study therefore identifies DesA1 as an immunodominant antigen with the potential to contribute to improved immunodiagnosis. 